\title{
Consideraciones farmacodinámicas y farmacocinéticas en los tratamientos habituales del paciente gerodontológico
}

\section{Farmacocinetic and farmacodinamic considerations in habitual treatment of gerontologic patient}

\author{
Arriola Riestra I*, Santos Marino J**, Martínez Rodríguez N***, \\ Barona Dorado C****, Martínez-González JM*****
}

\section{RESUMEN}

En los últimos años se está produciendo un aumento progresivo de la proporción y el número de personas mayores de 65 años. La gran mayoría de estas personas padecen a menudo algún tipo de enfermedad crónica y a menudo consumen uno o varios fármacos para tratarlas o disminuir sus síntomas. Por tanto debemos estudiar los cambios que se producen en el organismo durante la edad anciana y de que manera afectan a la farmacocinética y farmacodinamia de los medicamentos más utilizados en las consultas odontológicas..

Palabras clave: Gerodontología, antibióticos, analgésicos, anestésicos locales, antiinflamatorios, farmacocinética, farmacodinamia.

\section{SUMMARY}

In the last years a progressive increase of the proportion and the number of greater people of 65 years is taking place. The great majority of these people often suffers some type of chronic disease and often they consume one or several drugs to treat them or to diminish its symptoms. Therefore we must study the changes that take place in the organism during the old age and how they affect the farmacocinétic and farmacodinamic of medicines more used in our clinics.

Key words: Gerodontology, antibiotics, analgesics, anesthesics, antinflamatories, farmacocinetic, farmacodinamic.

Fecha de recepción: 19 de mayo 2008.

Aceptado para publicación: 2 de junio 2008.

* $\quad$ Odontólogo. Especialista en Implantoprótesis UCM.

** Odontólogo. Máster en Cirugía Bucal.

*** Alumno $5^{\circ}$ Curso Odontología.

**** Profesora Asociada de Cirugía Bucal UCM.

***** Profesor Titular de Cirugía Bucofacial UCM.

Arriola Riestra I, Santos Marino J, Martínez Rodríguez N, Barona Dorado C, Martínez-González JM. Consideraciones farmacodinámicas y farmacocinéticas en los tratamientos habituales del paciente gerodontológico. Av. Odontoestomatol 2009; 25 (1): 29-34. 


\section{INTRODUCCIÓN}

En los últimos años se está produciendo en la población mundial y especialmente en la española un aumento progresivo de la proporción y el número de personas mayores de 65 años. Ello es debido a la cada vez más alta esperanza de vida. Más de la mitad del mercado mundial de medicamentos está destinado a este grupo etario. La gran mayoría de estas personas padecen a menudo algún tipo de enfermedad crónica y a menudo consumen uno o varios fármacos para tratarlas o disminuir sus síntomas (1).

Los problemas ocasionados por el uso de fármacos en los ancianos, están relacionados con las interacciones entre ellos, así como los aspectos fisiológicos del envejecimiento, las enfermedades sistémicas, la nutrición y los aspectos psicológicos (2).

Se define envejecimiento como las modificaciones tanto biológicas, como morfológicas, bioquímicas y sociológicas que aparecen como consecuencia del tiempo en los seres vivos. Estas modificaciones, se desarrollan de manera distinta entre unos individuos y otros y de manera distinta entre los órganos de un mismo individuo. Esta variabilidad, influye en la farmacoterapia ya que no se debe utilizar de forma sistemática una posología concreta que sería insuficiente para unos pacientes y excesiva para otros (3).

Los ancianos presentan en general, una mayor incidencia de enfermedad, más cantidad de enfermedades simultáneas, mayor tendencia a la cronificación y/o invalidez, mayor dificultad en el diagnóstico de enfermedades (menor sintomatología), más necesidad de tratamiento rehabilitador, más cuidados psíquicos y/ o sociales, y como consecuencia de todo ello, mayor dificultad para establecer la farmacoterapia adecuada por el uso de múltiples fármacos, errores en su administración, etc. $(4,5)$.

Los profesionales de la Odontología, como clínicos que atendemos a los ancianos, debemos reducir los riesgos y aumentar los beneficios de la terapia farmacológica, comprendiendo los cambios farmacocinéticos y farmacodinámicos relacionados con la edad y las enfermedades más frecuentes relacionadas con este grupo de población.
El objetivo de este artículo es estudiar los cambios que se producen en el organismo durante la edad anciana y de que manera afectan a la farmacocinética y farmacodinamia de los medicamentos más utilizados en las consultas odontológicas.

\section{CONSIDERACIONES FARMACODINÁMICAS}

Se define farmacodinamia, como el estudio de los efectos bioquímicos y fisiológicos de los fármacos así como su mecanismo de acción. Analiza la interacción de la molécula del fármaco con el receptor. $(6,7)$

No disponemos de mucha literatura sobre las modificaciones farmacodinámicas en el anciano, pero algunos estudios han demostrado que en la vejez, los receptores sufren modificaciones tanto en su número como en su sensibilidad y la respuesta celular, así como otras modificaciones en el sistema central, periférico y autónomo en algunas de las enfermedades que afectan a estas edades, modificándose la respuesta a determinados medicamentos. Así, Cook (8) observó en 1984, que por alteración de los receptores, hay una mayor sensibilidad demostrada a fármacos que actúan sobre SNC como sedantes, benzodiacepinas y analgésicos potentes. Según Nolan y cols. (9), una menor cantidad de receptores beta-adrenérgicos y menor síntesis de AMPc, provocan una menor respuesta a fármacos estimuladores y bloqueantes de estos receptores y Feely (10) estudió en 1990 las alteraciones en la termorregulación y estabilidad postural, que afectan a fármacos con efectos sobre las mismas, como fenotiacinas, benzodiacepinas, antidepresivos tricíclicos, etc. También se ha visto que la enfermedad degenerativa vascular en ancianos induce una respuesta hemostática menor, así como disminución de factores de coagulación y aumento de fragilidad capilar, aumentando así el riesgo de hemorragia tras la administración de fármacos anticoagulantes.

\section{CONSIDERACIONES FARMACOCINÉTICAS}

La farmacocinética es el estudio de la absorción, distribución, metabolismo y excreción de los medicamentos. Todos estos procesos están alterados en 
mayor o menor medida en el anciano. La farmacocinética sugiere que los cambios en la respuesta a medicamentos con la edad se deben a estas alteraciones.

La absorción enteral de un fármaco está determinada por su solubilidad, la dosis, el flujo sanguíneo, y la motilidad gastrointestinal.

A partir de los 65 años, se producen cambios morfológicos y fisiológicos que pueden modificar la absorción de un medicamento, tales como: aumento del pH gástrico, disminución de la producción de ácido gástrico, disminución de la velocidad de vaciamiento gástrico, disminución del flujo sanguíneo esplácnico, disminución de la motilidad intestinal, y disminución de la superficie de absorción.

Sin embargo, la mayoría de los fármacos utilizados en la práctica odontológica, son absorbidos por difusión pasiva durante el contacto con la superficie para la absorción del intestino delgado, por tanto los factores anteriores, no parecen evidencia suficiente para explicar la disminución en la absorción.

Una posible causa sería la interacción de los fármacos con la comida ya que la ausencia de alimentos en el intestino mejora la absorción de algunas drogas como los antibióticos, pero es distinto con otras.

En el anciano, por acción de los medicamentos, hay una disminución en la salivación que implica dificultad en la deglución, y disminución de la disolución de medicamentos administrados por vía sublingual.

Con respecto a la administración de fármacos por vía intramuscular, debemos considerar la menor proporción de masa muscular y de flujo sanguíneo en el paciente anciano.

En relación a la distribución, una vez que el fármaco está absorbido se vehiculiza a todo el organismo aprovechando la corriente sanguínea. Este transporte del fármaco por vía sanguínea desde el lugar de entrada lo lleva al líquido extravascular, de modo reversible (distribución) o irreversible (eliminación).
Con el envejecimiento se producen cambios que afectan a la distribución de los fármacos como:

- Modificaciones de la composición corporal: aumento del tejido adiposo, disminución de la masa músculo esquelético, disminución del agua corporal total, disminución del agua extracelular.

- Modificaciones en las proteínas plasmáticas e hísticas: disminución de la albúmina, aumento de la alfa-glicoproteína.

- Otras modificaciones corporales: disminución del gasto cardíaco, aumento de las resistencia vascular periférica, disminución del gasto sanguíneo hepático y renal y aumento de la fracción de eyección cardíaca hacia la circulación cerebral, la coronaria y la de músculo esquelético.

Factores como la disminución del peso corporal, del agua, de la albúmina, del riego de los órganos, del rendimiento cardíaco y aumento de los depósitos grasos, pueden dar lugar a que fármacos liposolubles como la lidocaína y las benzodiacepinas tengan un volumen de distribución aumentado y se acumulen en mayor proporción en el tejido graso. Por el contrario fármacos hidrosolubles como el paracetamol, tienen menor volumen de distribución y alcanzan niveles plasmáticos que en el adulto joven, por lo que es importante ajustar las dosis de los fármacos de acuerdo a las modificaciones en el volumen de distribución de cada principio activo.

El concepto de metabolismo o biotransformación, se define como los cambios que se producen en las moléculas de los fármacos mediante la acción de sistemas enzimáticos que se encuentran localizados en distintos lugares del organismo, principalmente el hígado.

En el anciano se producen una serie de cambios fisiológicos que pueden modificar el metabolismo farmacológico, tales como: disminución del flujo sanguíneo hepático, disminución de la masa hepática (menos hepatocitos funcionantes) y disminución de la capacidad metabólica hepática.

Esta disminución es variable de un medicamento a otro y de un individuo a otro y determina una mayor vida media de algunas drogas en el organismo, por 
enlentecimiento de su metabolismo como se comprobó con la lidocaína.

El comportamiento de las drogas va a depender además, del consumo de tabaco y alcohol ya que afectan al metabolismo hepático.

Los cambios producidos por la edad pueden perjudicar a la fase I del metabolismo (oxidación, reducción e hidrólisis); mientras que la fase II (glucoronización, acetilación y sulfación) se verá menos afectada.

Con relación a la excreción, la vía más importante de eliminación de los fármacos es la renal. Greenblatt y cols. (11) observaron en 1982 que es a este nivel donde se producen los efectos más notables de la edad sobre la farmacocinética de los medicamentos. Las modificaciones fisiológicas que se producen en los ancianos y que podrían afectar a la eliminación renal de los fármacos, incluyen: disminución del flujo sanguíneo renal, disminución de la filtración glomerular y disminución de la capacidad de secreción tubular renal.

La función renal se evalúa con un "clearance" de creatinina sérica que disminuye $1 / 3$ entre los 20 y los 90 años, aunque se ha visto que esto es muy variable de unos individuos a otros.

La disminución de la filtración glomerular y la función tubular renales, es el principal factor que modifica la semivida plasmática de un gran número de fármacos. Este hecho, no es tan importante en fármacos que presentan un margen terapéutico amplio como penicilinas y cefalosporinas, en las que valores plasmáticos elevados pueden carecer de importancia clínica en el anciano, pero es de vital importancia en aquellos fármacos que se eliminan fundamentalmente por vía renal y que presentan un estrecho margen terapéutico.

En casi todas las situaciones, puede ser conveniente usar el método de ajuste por intervalos: no cambiar la dosis, pero se amplía el periodo entre su administración en proporción a la reducción de la insuficiencia renal.

Por tanto, en el anciano debemos determinar la disminución de la función renal para evitar sobredosis relativas, excesiva acumulación del fármaco, efectos adversos concomitantes, y una semidesintegración prolongada, como ya enunció Terezhalmy (12) en 1989.

\section{CONSIDERACIONES SOBRE LOS FÁRMACOS MÁS UTILIZADOS EN ODONTOGERIATRÍA}

\section{Analgésicos}

- Opiáceos: Son poco utilizados en odontología debido a que podemos conseguir una analgesia adecuada en la mayoría de los casos con un analgésico no opiáceo evitando así efectos indeseables como convulsiones, depresión respiratoria y colapso cardiovascular. En su caso utilizaremos siempre codeína a bajas dosis asociada a un AINE.

- No opiáceos: Será el analgésico de elección pero debemos tener en cuenta que pueden producir reacciones adversas especialmente en pacientes mayores de 65 años sobre todo de tipo gástrico y hematológico, sobre todo en al caso de el ácido acetilsalicílico que también pueden producir toxicidad renal, y algunos como el paracetamol a elevadas dosis puede producir incluso necrosis hepática. Debemos tener precaución a la hora de combinarlos con fármacos como los anticoagulantes ya que va a aumentar su efecto y los antihipertensivos y antidiuréticos que lo van a disminuir. Por sus pocos efectos adversos y su capacidad antipirética y analgésica, el Paracetamol es el analgésico de elección para pacientes mayores de 65 años con dolores de tipo leve o moderado.

\section{Antiinflamatorios}

- No Esteroideos (AINE): cuando se administran de forma adecuada, además de tener actividad analgésica, actúan como antiinflamatorios de efecto duradero. Inhiben la ciclooxigenasa disminuyendo la inflamación, últimamente se prefieren los inhibidores de la ciclooxigenasa-2, pero se ha estudiado que pueden agravar problemas de tipo cardiovascular, por lo que debemos utilizarlos solo en pacientes con riesgo de úlcera o 
problemas gástricos y siempre y cuando tengamos claro que no tienen patologías cardiovasculares (13). Son los más utilizados y debemos tener en cuenta consideraciones similares a las del caso de los analgésicos de tipo no opiáceo, pueden provocar nefritis en casos de insuficiencia renal, y son antiagregantes plaquetarios, por lo que debemos tener precaución en pacientes mayores de 65 años.

- Corticoides: habitualmente, no son muy utilizados en la consulta odontológica (su uso está prácticamente restringido al campo de la cirugía), pero debemos tener en cuenta que pueden producir HTA, osteoporosis, atrofia muscular y suprarrenal y síndrome de Cushing y debemos evitar su uso en pacientes hipertensos, anticoagulados, diabéticos y con procesos infecciosos.

\section{Antibióticos}

Según la última revisión de la GPT (13), se recomienda utilizar penicilinas como primera opción seguidos de imidazoles y quinolonas, en cualquier caso haremos un breve repaso sobre todos los antibióticos más utilizados.

- Betalactámicos: Van a ser en general la primera elección debido a su buena tolerancia y baja toxicidad pero deberemos recordar que su vida media plasmática se ve aumentada en pacientes con insuficiencia renal y pueden aumentar el tiempo de trombina y protrombina.

- Macrólidos: No se han descrito cambios farmacocinéticos relacionados con la edad, por lo que van a ser una buena alternativa a los betalactámicos.

- Quinolonas: Son también bastante seguras, debido a su amplio espectro antimicrobiano, su buena absorción en el tracto gastrointestinal, su excelente distribución en los tejidos y sus escasas reacciones adversas.

- Clindamicina: Debemos tener en cuenta, sobre todo, el riesgo de producir colitis pseudomembranosa en ancianos.

- Tetraciclinas: Pueden agravar la insuficiencia renal.

- Metronidazol: Se metaboliza en hígado y su excreción es renal, por lo que sus niveles séricos pueden aumentar considerablemente en ancianos y algunos autores recomiendan reducir sus dosis hasta un 30-40\% (14).

\section{Anestésicos locales}

Debido a los cambios producidos en el organismo del paciente geriátrico antes comentados, van a disminuir su metabolismo y excreción aumentado su vida media por lo que debemos ajustar las dosis para reducir la toxicidad.

- Lidocaína: Es el anestésico local más comúnmente utilizado en la consulta odontológica, pero debido a su poder vasodilatador debemos asociarlo a un vasoconstrictor y es ahí donde pueden aparecer las complicaciones por lo que debemos tener cuidado con pacientes cardiópatas (15). No combinar con antiarrítmicos, B-bloquentes, diuréticos y miorrelajantes.

- Mepivacaína: Será una buena elección en odontogeriatría debido a que por su escaso poder vasodilatador, no será necesario asociarlo a un VC. La tendencia a la hemorragia aumenta en combinación con AINES, y heparinas y aumenta los efectos de los miorrelajantes.

- Prilocaína: También será una buena elección debido a la corta duración de su efecto y su baja toxicidad. Si bien debemos tener en cuenta que es el único anestésico local que combina un metabolismo hepático con un metabolismo pulmonar por lo que debemos evitar su uso en pacientes con problemas a ese nivel (16). Puede producir depresión miocárdica si interacciona con antiarrítmicos y en combinación con sulfamidas aumenta el riesgo de metahemoglobinemia.

- Articaína: Si bien es un anestésico muy utilizado en odontología debemos restringir su uso en pacientes $>65$ años debido a la larga duración de su efecto y al riesgo de metahemoglobinemia, su uso está contraindicado en insuficiencia cardíaca y/o renal.

- Bupivacaína: También debemos evitar su uso por la muy larga duración de su efecto y el elevado riesgo de cardiotoxicidad (17). Aumentan el riego de depresión cardíaca con antiarrítmicos y Bbloqueantes. 


\section{CONCLUSIONES}

Todos los procesos farmacocinéticos están modificados en mayor o menor medida en el paciente mayor de 65 años (18), por lo que debemos ser cautelosos a la hora de prescribir cualquier medicamento teniendo en cuenta las posibles interacciones y patologías para evitar reacciones adversas evaluando siempre el riesgo y el beneficio de cada uno de ellos.

\section{BIBLIOGRAFÍA}

1. Instituto Nacional de Asuntos Sociales. La Tercera Edad en España: aspectos cuantitativos. Madrid: Ministerio de Asuntos Sociales; 1989.

2. García A, Horga J y cols. Prescripción racional de fármacos. Madrid: Médic S.A Edimisa; 1990. p. 15-206.

3. De Paz Díaz A, Guerra Polo J. Yatrogenia y uso de fármacos. Guillén Llera F, Pérez del Molino Martín J. Síndromes y Cuidados en el Paciente Geriátrico. España: Masson; 1994. p. 239-47.

4. Bullón Fernández P, Velasco Ortega E. Odontoestomatología geriátrica. Madrid: IMEC; 1996.

5. Hol-Pedersen P, Löe H. Textbook of Geriatric Dentistry. Copenhagen: Munksgaard; 1986.

6. Bascones Martínez A. Tratado de Odontología, Tomo IV. Madrid: Trigo; 1998. p. 3583-600.

7. Bullón Fernández P, Machuela Portillo G. Tratamiento odontológico en Pacientes Especiales. Madrid: Normon; 2004.

8. Cook PJ, Flanagan R, James IM. Diazepam tolerance: Effects of age, regular sedation and alcohol. B Med Clin Res. 1984 289:351-3.

9. Nolan I, O’Malley K. Prescribing for the elderly. Part I: Sensivity of the elderly to adverse drug reaction. J American Geriatric Society. 1980 36:142-9.

10. Feely J, Coakley D. Altered Pharmacodynamics in the elderly. Clinical Geriatric Medicine. 1980; Jun 6:269-83.

11. Greenblatt DJ. Reduced serum albumin concentration in the elderly: A report from the Boston Collaborite Drug Survillance Program. American Geriatric Society. 1979;27:20-32.

12. Terezhalmy GT. Farmacoterapia racional en la vejez. Clínicas Odontológicas de Norteamérica. 1989;1:61-8.

13. Guía de Prescripción Terapéutica, Agencia Española de Medicamentos y Productos Sanitarios. Pharma Editores S.L. 2007.

14. Bascones A. Diagnóstico y tratamiento: Infecciones odontógenas periodontales y periimplantarias. Madrid: Drug Farma S.L. 1996.

15. Gay Escoda C, Berini Aytes L. Anestesia Odontológica. Madrid: Avances. 1997.

16. Peñarrocha M, Martínez-González, JM, Sanchís Bielsa JM. Anestesia local en Odontología. Valencia: Promolibro. 1999.

17. García-Peñín A, Guisado-Moya B, Montalvo-Moreno J. Riesgos y complicaciones de anestesia local en la consulta dental. Estado actual. RCOE. 2003;8(1):67-71.

18. García Fj. Características de las enfermedades en Geriatría. Salgado A, Guillén F. Manual de Geriatría. Barcelona: Salvat. 1990;157-63.

\section{CORRESPONDENCIA}

Ignacio Arriola Riestra

drnacus@hotmail.com 